\title{
Assistência em saúde prestada à criança com diagnóstico de microcefalia, em um município do interior da Amazônia
}

\section{Assistance children diagnosed with microcefalia in the municipality of the interior of the Amazon}

\author{
1 Maria do Socorro Almeida do Nascimento \\ 1 Paula Malena Ribeiro Batista \\ 2 Maria da Conceição Cavalcante Farias \\ 3 Luana Almeida dos Santos luanah.orix@gmail.com \\ ${ }^{4}$ Nádia Vicência do Nascimento Martins
}

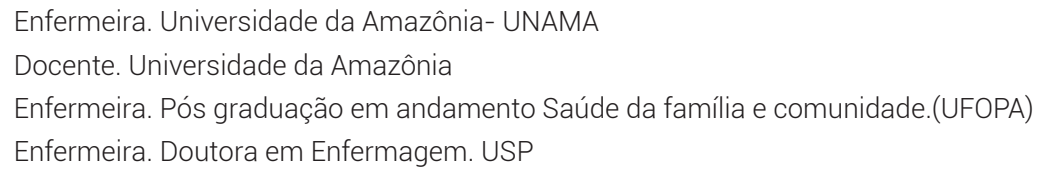

\section{Resumo}

A microcefalia tem sido alvo de constante discussão entre os profissionais de saúde, após frequentes notificações de crianças nascidas com essa malformação congênita. Dessa forma, esta pesquisa teve por objetivo conhecer a assistência em saúde prestada a crianças nascidas com microcefalia nos serviços de saúde em Santarém-Pará. Trata-se de uma pesquisa exploratória, com abordagem quantitativa, em que foi utilizado um questionário com respostas fechadas, aplicado a usuários e profissionais de saúde que atuam diretamente com portadores de microcefalia. A presente pesquisa revelou que $100 \%$ (10) das entrevistadas (usuárias) realizaram pré-natal. Destas, $80 \%$ (8) só descobriram a microcefalia após o parto. As famílias recebem atendimento no Centro de Referência à Saúde da Criança e contam com a participação de profissionais como: enfermeiro, médico, fisioterapeuta, terapeuta ocupacional e fonoaudiólogo. Na pesquisa, foi possível perceber que alguns pontos devem ser reorganizados, para que haja um atendimento de qualidade a crianças com malformação congênita, pois, por menores que sejam as modificações, elas podem contribuir com a melhoria da qualidade de vida tanto das mães quanto das crianças com essa malformação.

Palavras-chave:

Microcefalia. Assistência em saúde. Enfermagem.

\begin{abstract}
Currently, microcephaly has been the subject of constant discussion among health professionals, following the frequent reports of children born with the disease during the last months. know the assistance provided in the health services available for the monitoring of children diagnosed with microcephaly in Santarém-Pará. This is a quantitative and exploratory research with the application of a questionnaire with closed questions, made available to users and health professionals who work directly with patients with microcephaly. The present research revealed that 100\% (10) of the interviewed (users) performed prenatal care, of these $80 \%$ (8) only discovered microcephaly after delivery. The families receive care at the Reference Center for Child Health and have the participation of nurses, physicians, physiotherapists, occupational therapists and speech therapists. In view of the present research, it was possible to perceive that some points among the care must be reorganized so that there is a quality care to the congenital malformation bearers, since small details can interfere directly in the quality of life of the bearer with the disease.
\end{abstract}

\section{Keywords:}

Microcephaly. Care. Nursing.

\section{Como você deve citar?}

SANTOS, Luana Almeida dos et al. Assistência em saúde prestada à criança com diagnóstico de microcefalia, em um município do interior da Amazônia. Cadernos UniFOA, Volta Redonda, n. 39, p. 95 -105, abril. 2019. 


\section{INTRODUÇÃO}

A microcefalia é uma condição neurológica, associada à redução do perímetro cefálico e do cérebro. De acordo com o Ministério da Saúde, houve uma alteração da medida determinante do perímetro cefálico para diagnóstico de microcefalia, que passou a ser $31,9 \mathrm{~cm}$ para menina e $31,5 \mathrm{~cm}$ para meninos. O propósito da alteração é padronizar as referências cefálicas para todos os países, valendo para bebês nascidos com 37 ou mais semanas de gestação (PERUCCI, 2015; BRASIL 2016).

Percebe-se que a maioria das crianças acometidas pela microcefalia apresentam graves problemas relacionados à malformação do sistema nervoso, dificuldade no sistema muscular e motor, ocasionando, assim, um déficit no desenvolvimento físico e psicomotor.

De acordo com dados informados pelo Ministério da Saúde (2016), o número de casos de Microcefalia no Brasil aumentou significativamente no ano de 2015 em todo país. Dentro do sistema de saúde, há uma declaração de nascido vivo na qual consta a malformação congênita. Porém, muitas vezes, a declaração de nascido vivo não é preenchida corretamente, gerando a omissão de informação em determinados casos (BRASIL, 2016).

Salienta-se que os casos de microcefalia estão sendo considerados como um problema de saúde pública em todo o mundo, haja vista que a doença compromete consideravelmente o desenvolvimento da criança, limitando assim suas atividades diárias. Diante disso, vários estudos vêm sendo realizados em prol de inibir a proliferação da infecção do Zika Vírus, especificamente em gestante, com intuito de evitar casos de microcefalia relacionado à infecção.

Fischer (2016) menciona que, de acordo com a Secretaria de Estado de Saúde do Pará (SESPA), no ano de 2015, foram registrados 15 casos de microcefalia no estado e Guillain-Barré, em 2016, apenas três casos até fevereiro do presente ano. Frisa-se que nenhum desses números teve qualquer associação com o vírus do Zika.

A microcefalia apresenta como fator de risco na maior parte dos casos, a infecção da mãe durante a gestação por: toxoplasmose, rubéola e citomegalovírus. Normalmente, essa contaminação acontece no primeiro trimestre gestacional, período em que a região cerebral do embrião está em processo de formação. No entanto, não se pode descartar a influência do abuso de álcool e drogas ilícitas na formação e desenvolvimento do feto, assim como a ligação com o Zika vírus (JANSEN, 2016).

Reforça-se que as causas da doença transmitida à criança podem estar relacionadas com as complicações durante o parto que venham ocasionar a diminuição na oferta de oxigênio ao feto, mulheres que se expõem a produtos químicos durante o período gestacional, desnutrição grave, fenilcetonúria, assim como contaminação por rubéola, toxoplasmose e citomegalovírus.

Estudos recentes demostram que a microcefalia pode ter ligação com o vírus Zika, que, por possuir sintomas mais brandos que os da Dengue e os da febre Chicungunya (doenças transmitidas pelo mosquito Aedes aegypti), chegou a ser relevada pelas autoridades de saúde, no entanto, atualmente estudos indicaram uma associação do vírus Zika a casos de microcefalia congênita e a síndrome Guillain-Barré (BRASIL, 2016).

De acordo com Perucci (2015), o diagnóstico dessa anomalia pode ser realizado durante a gestação, por meio de exames durante a assistência pré-natal, confirmado logo após o parto com a medição do perímetro cefálico. Exames complementares como a tomografia computadorizada ou ressonância magnética auxiliam para verificação do quadro e quais as possíveis consequências para o desenvolvimento do bebê. 
Salienta-se que o pré-natal, quando realizado de forma correta, pode detectar diversos problemas relacionados ao infante ainda no ventre da mãe, sendo possível realizar o tratamento com o intuito de minimizar o sofrimento do bebê ao nascimento.

O principal sintoma da microcefalia é a redução da circunferência da cabeça da criança, gerando uma hipertrofia muscular acompanhada de crises convulsivas, paralisia e atraso mental, o que compromete consideravelmente o desenvolvimento da criança (BRASIL, 2016).

É de suma importância que o profissional de enfermagem entenda seu papel, na orientação e suporte aos cuidados com as crianças com necessidades especiais de saúde. O processo de cuidado inclui, além da criança com microcefalia, os familiares ou outras pessoas que participam da identificação e atendimento das necessidades humanas, considerando também o sentimento. A enfermagem no cuidado à criança envolve interação, vinculo, aconselhamento e sobretudo apoio à pessoa responsável pelo cuidado (SANTOS, et al. 2010).

Diante desse contexto, o presente trabalho tem como objetivo conhecer a assistência em saúde prestada a crianças nascidas com microcefalia nos serviços de saúde em Santarém-Pará. 0 interesse em desenvolver esta pesquisa se deve ao fato de que crianças diagnosticadas com microcefalia necessitam de um acompanhamento de qualidade e um atendimento especializado, promovendo melhor qualidade de vida. A microcefalia é uma doença que não tem cura e, após o quadro da doença instalado, a única alternativa são as terapias que visam melhorar a qualidade de vida da criança, estimulando suas possibilidades de desenvolvimento motor, intelectual e emocional.

Ressalta-se ainda que as terapias de suporte devem estar à disposição da criança, pois, quanto mais cedo diagnosticada a doença e iniciado o tratamento, mais chances a criança tem de reduzir as sequelas da microcefalia. A fonoaudiologia, a terapia ocupacional e principalmente a enfermagem e fisioterapia são primordiais para o sucesso na melhora do quadro clínico.

\section{MATERIAL E MÉTODO}

Trata-se de um estudo exploratório e descritivo com delineamento quantitativo, realizado no Centro de Referência em Saúde da Criança do município de Santarém - Pará, serviço de saúde destinado a prestar assistência a pacientes com microcefalia da região oeste do Pará e municípios vizinhos.

O centro de referência oferece consultas especializadas por intermédio de uma equipe multidisciplinar, composta por: pediatra, enfermeiro, fisioterapeuta, terapeuta ocupacional, fonoaudiólogo, psicólogo e assistente social, ofertando atendimentos a crianças com alto grau de debilidade, buscando proporcionar uma atenção integral à criança e seus familiares.

A pesquisa foi realizada no Centro de Referência à Saúde da Criança, no período de janeiro de 2017 a maio de 2017, no qual foram identificados dez (10) pacientes com malformação congênita, com registro de acompanhamento regular no Centro de Referência.

Foram inclusos na pesquisa os pais e ou responsável legal pela criança com microcefalia em processo de acompanhamento no Centro de Referência e os profissionais de saúde que atuam no serviço e que aceitaram participar voluntariamente da pesquisa mediante assinatura do Termo de Consentimento Livre e Esclarecido/TCLE, totalizando cinco (5) profissionais. 
Os critérios de exclusão foram os profissionais, pais e responsáveis legais que obtiveram diagnóstico de microcefalia fora do período de análise, que residem em municípios vizinhos, que solicitaram a transferência para continuidade do tratamento em outra cidade ou serviço de saúde ou com diagnóstico sem referência à temática em questão.

Mediante a apresentação do projeto e seus objetivos, foi apresentado e lido o Termo de Consentimento Livre e Esclarecido (TCLE), que, somente após a aceitação por meio da assinatura, a coleta de dados foi iniciada. 0 questionário que foi aplicado aos pais/responsáveis da criança com malformação congênita contém nove (9) questões de múltipla escolha voltadas para os cuidados com o infante.

A coleta de dados foi realizada por meio de questionário, prontuários e livro de registro. 0 estudo contou com a utilização de dois questionários, um aplicado aos pais ou responsável legal da criança com microcefalia e o outro direcionado aos profissionais de saúde que atuam diretamente no atendimento dessas crianças.

O mesmo processo foi adotado na pesquisa com os profissionais de saúde do Centro de Referência à Saúde da Criança, que contou com aplicação de um questionário contendo cinco (5) questões de múltipla escolha sobre satisfação do colaborador que atua diretamente com o portador de microcefalia, que também foi aplicado após a leitura, aceitação e assinatura do Termo de Consentimento Livre e Esclarecido (TCLE).

A pesquisa foi desenvolvida em duas etapas. Na primeira etapa, foram abordadas as seguintes temáticas: nível de satisfação do profissional com relação ao atendimento do paciente com microcefalia, com participação de 5 profissionais da saúde. No segundo momento, a temática foi focada em dados sociodemográficos; quadro clínico gestacional; compreensão da microcefalia; nível de satisfação do usuário, com a participação de 10 pais e/ou responsáveis legais de crianças acompanhadas com microcefalia.

Os resultados da pesquisa foram tabulados no programa Microsoft Word 2010, embasado em estatística descritiva, apresentado em tabelas para a melhor compreensão dos resultados. Após análise, houve a contextualização e discussão dos resultados.

A pesquisa está de acordo com os princípios que rege a Resolução 466/2012, que envolve as diretrizes e normas reguladoras de pesquisa direcionada a seres humanos, mediante autorização e aprovação da Secretaria Municipal de Saúde e a aprovação do projeto no Comitê de Ética e Pesquisa da Universidade do Estado do Pará- Campus XII- Tapajós, conforme o Parecer de número: 1.865.776.

\section{RESULTADOS E DISCUSSÃO}

Os resultados representam o nível de satisfação da população com relação ao atendimento ofertado às crianças diagnosticadas com microcefalia atendidas no Centro de Referência à Saúde da Criança, no município de Santarém, Pará.

A tabela 1 mostra a assistência prestada pelos profissionais de saúde no atendimento às crianças com microcefalia. Nela, é possível perceber que $60 \%$ (3) dos profissionais de saúde expressaram dificuldade em realizar o atendimento, mencionando que a falta de capacitação e atualização, tal como carência de equipamentos e insumos, são os responsáveis pelo atendimento deficiente. 
Porém, mesmo com todas as barreiras encontradas pelos profissionais dentro do sistema de saúde, ainda assim $100 \%$ (10) dos colaboradores informaram que orientam a família com relação à patologia e sobre a importância do acompanhamento da criança.

A pesquisa de Barcellos e Rapopori (2014) relata que os indivíduos que apresentam qualquer tipo de deficiência, seja ela física ou mental, necessitam de um atendimento especializado com profissionais qualificados e capacitados para instruir adequadamente o paciente e seus familiares sobre os cuidados e a evolução da doença.

É importante destacar que o serviço de qualidade acontece quando há um ambiente de trabalho adequado e um profissional capacitado para tal função, haja vista que o tratamento do paciente com microcefalia deve acontecer durante toda a vida. Entretanto, devem ser estabelecidas metas e normas de cuidados que possam facilitar o tratamento, buscando-se sempre a melhoria na qualidade de vida (NORBERT, et al. 2016).

Tabela 1 - Assistência em saúde prestada por profissionais de saúde a crianças com microcefalia, acompanhadas no Centro de Referência à Saúde da Criança, em Santarém, Pará.

\begin{tabular}{lll}
\hline Descrição & $\mathbf{n}=\mathbf{5}$ & $\%$ \\
\hline Tipos de atendimentos disponíveis aos pacientes com microcefalia & & \\
\hline Enfermeiro & 01 & $20 \%$ \\
\hline Médico & 01 & $20 \%$ \\
\hline Fisioterapeuta & 01 & $20 \%$ \\
\hline Terapeuta Ocupacional & 01 & $20 \%$ \\
\hline Fonoaudiólogo & 01 & $20 \%$ \\
\hline Dificuldade no atendimento as crianças com microcefalia & \\
\hline Sim & 03 & $60 \%$ \\
\hline Não & 02 & $40 \%$ \\
\hline Cuidado com orientação aos cuidadores sobre o que é a microcefalia & \\
\hline Sim & 05 & $100 \%$ \\
\hline Informação os cuidadores sobre o acompanhamento pela equipe de saúde & \\
\hline Sim & 05 & $100 \%$ \\
\hline
\end{tabular}

Fonte: próprios autores, 2019.

O perfil sociodemográfico das famílias entrevistadas revelou que $60 \%$ (6) dos pais ou responsáveis pelas crianças com microcefalia encontram-se com idade entre 15 e 25 anos; $20 \%$ (2) apresentavam-se na faixa etária de 26 a 35 anos; e o equivalente a $20 \%$ (2), com idade entre 36 a 45 anos. Quando relacionado à escolaridade dos entrevistados, $40 \%$ (4) declararam que possuem ensino médio incompleto e $60 \%$ (6) apresentam ensino médio completo.

Diante do perfil sociodemográfico dos familiares apresentado, é possível perceber certa maturidade para o cuidado de crianças com microcefalia, levando em consideração a faixa etária e escolaridade, facilitando a compreensão das informações ofertadas por profissionais de saúde aos pais ou responsáveis por tratar e acompanhar a criança. Essa constatação apresenta-se em conformidade com a orientações expostas na pesquisa de Sousa et al. (2010).

Reforça-se que uma família estruturada, configura-se como alicerce dentro do contexto familiar, pois somente com apoio e compreensão de todos os atores familiares é possível unir forças para enfrentar os desafios da vida. 
Tabela 2 - Perfil sociodemográfico dos pais e/ou responsáveis de crianças com microcefalia acompanhadas no Centro de Referência à Saúde de Santarém-Pará.

\begin{tabular}{lll}
\hline Descrição & $\mathbf{n = 1 0}$ & \% \\
\hline Faixa etária & & $60 \%$ \\
\hline 15 a 25 anos & 06 & $20 \%$ \\
\hline 26 a 35 anos & 02 & $20 \%$ \\
\hline 36 a 45 anos & 02 & \\
\hline Escolaridade & & $40 \%$ \\
\hline Ensino Médio incompleto & 04 & $60 \%$ \\
\hline Ensino Médio completo & 06 &
\end{tabular}

Fonte: próprios autores, 2019.

A tabela 3 destaca informações sobre a história obstétrica das mães de crianças com microcefalia. Com esses resultados, é perceptível que 50\% (5) declararam até duas (2) gestações; $40 \%$ (4), apenas uma (1) gestação; e que 10\% (1), três (3) ou mais gestações.

Dessas mulheres, apenas 10\% (1) disseram ter tido filho com malformação congênita em gestações anteriores. Quando questionadas sobre a realização de pré-natal, 100\% (10) disseram ter recebido acompanhamento pré-natal e, destas, $90 \%$ (9) relataram ter realizado todos os exames laboratoriais, e 10\% (1) não souberam informar.

É necessário compreender que a quantidade de gestações não é fator de predisposição para problemas voltados à malformação congênita, porém fatores como: doenças genéticas que possam gerar defeito no crescimento e desenvolvimento cerebral; uso de álcool, drogas e substâncias químicas durante a gestação; desnutrição na gestação; infecção do sistema nervoso central com algum tipo de doença, como a rubéola, toxoplasmose, citomegalovírus, zika vírus, dentre outras, podem ser fundamentais para o comprometimento do desenvolvimento saudável na gestação (MENEZES et al., 2016; FILHO et al., 2005).

Destaca-se que são diversas as causas de microcefalia e que sua detecção precoce pode ocorrer durante a assistência pré-natal. Por conta isso, é importante o pré-natal de qualidade para as gestantes, buscando-se identificar riscos e problemas relacionados à mãe e ao bebê, com intuito de evitar ou ao menos minimizar possíveis sequelas.

Os resultados destacados na pesquisa de Nader et al. (2007) corroboram com esta pesquisa, quando relatam que, durante o pré-natal, é possível prevenir e detectar diversas patologias que são responsáveis por taxas de mortalidade materno-infantil.

Na pesquisa realizada por Costa et al., (2010) fica evidente que a assistência ao pré-natal deve ser realizada através de cuidados minuciosos voltados a todo o processo gravídico-puerperal que envolve desde as transformações fisiológicas até as mudanças psicológicas, porém é necessário que o profissional de saúde procure atender à gestante de forma holística, buscando perceber o que mais incomoda na gestação, porém também buscando investigar situações que, por vezes, se mostram assintomáticas, visando à identificação e tratamento, quando necessário, o mais precoce possível.

A Atenção Primária em Saúde é a porta de entrada para a investigação de futuros problemas relacionados com a mãe e o bebê durante o processo gestacional, haja vista que o pré-natal é o programa de saúde responsável por realizar a coleta de informações voltadas para o conhecimento do perfil demográfico, socioeconômico e clínico da mulher, abrangendo o histórico de gestações anteriores, 
identificação de intercorrências, problemas de saúde, dentre outros, possibilitando que o profissional de saúde possa realizar um diagnóstico do quadro clínico atual da gestante e, dessa forma, identificar e intervir em prováveis intercorrências, com intuito de evitar e ou minimizar danos.

É importante mencionar que qualquer alteração no processo gestacional poderá ocasionar uma deficiência no desenvolvimento embrionário e, consequentemente, resultar em anomalias congênitas que podem acarretar comprometimentos resultantes de problemas funcionais ou estéticos. É possível observar comprometimentos neurológicos, motores, malformações congênitas, dentre outros, ainda no período gestacional (SANTOS e DIAS, 2005; AMARAL et al., 2016).

O estudo realizado por Sousa et al. (2010) menciona que a malformação congênita é um problema que pode estar relacionado a diversos fatores como: condições socioeconômicas, idade da mãe, deficiências nutricionais, contato direto com radiações ionizantes, alcoolismo, rubéola, sífilis congênita, traumatismo, distúrbios genéticos e determinados fármacos utilizados durante a gravidez. Todos esses fatores podem interferir no processo de desenvolvimento do bebê e causar complicações no decorrer da gestação após o nascimento e, por isso, é importante o acompanhamento minucioso durante todo período gestacional.

Salienta-se que o período gestacional é um momento em que a mãe deve realizar diversos cuidados, seja com a saúde, alimentação, seja com realização de exames preventivos. Cada cuidado realizado pela mulher durante a gestação poderá prevenir o infante de graves problemas de saúde.

Tabela 3 - Histórico gestacional das mães de crianças com microcefalia atendidas no Centro de Referência em Saúde da Criança, Santarém-Pará.

\begin{tabular}{lll}
\hline Descrição & $\mathbf{n = 1 0}$ & $\%$ \\
\hline Número de gestações & 04 & $40 \%$ \\
\hline Uma (1) & 05 & $50 \%$ \\
\hline Duas (2) & 01 & $10 \%$ \\
\hline Três (3) & & \\
\hline Malformação congênita & 01 & $10 \%$ \\
\hline Sim & 09 & $90 \%$ \\
\hline Não & & \\
\hline Realização do Pré-natal & 10 & $100 \%$ \\
\hline Sim & & \\
\hline Realização exames obrigatórios & 09 & $90 \%$ \\
\hline Sim & 01 & $10 \%$ \\
\hline Não sabe & & \\
\hline
\end{tabular}

Fonte: próprios autores, 2019.

A tabela 4 apresenta o meio pelo qual a gestante e a família receberam o diagnóstico de microcefalia. De acordo com a pesquisa, 70\% (7) dos entrevistados declararam ter recebido o diagnóstico apenas após o nascimento, isto é, no pós-parto; e 30\% (3) informaram que as alterações e o diagnóstico foram detectados previamente, por meio do exame de ultrassonografia obstétrica. Quando questionado sobre o conhecimento acerca da microcefalia, 100\% (10) dos pais e/ou responsáveis informaram terem sido orientados por profissionais de saúde e declararam saber o conceito de microcefalia.

De acordo com Santos e Dias (2005), o conceito de malformação congênita está relacionado a toda anomalia funcional ou estrutural que acomete a criança desde o momento do nascimento e perdura por toda a vida. Porém, a microcefalia pode ser detectada ainda na gestação por meio de ultrassono- 
grafia, sendo esta, considerada atualmente como um exame imagem complementar, preconizado pela assistência pré-natal e reconhecido como direito da gestante durante o período gravídico. Seu avanço tecnológico tem se tornado algo essencial para a detecção de doenças congênitas, pois proporciona perfeição na visualização de modificações anatômicas anormais no feto ainda em desenvolvimento (OLIANI et al., 2015).

O ultrassom é uma das principais ferramentas utilizadas pelos profissionais de saúde durante o pré-natal, pois, é através dela que pode ser observado o feto na íntegra na fase intrauterina. Além da análise do bebê, ainda é possível perceber outras estruturas como: líquido amniótico, cordão umbilical, grau de amadurecimento placentário, dentre outros. (BARINI et al., 2002).

Tabela 4 - Conhecimento e forma de diagnóstico de microcefalia

\begin{tabular}{lll}
\hline Descrição & $\mathbf{N}=\mathbf{1 0}$ & $\%$ \\
\hline Deteç̧ão da Microcefalia & & $30 \%$ \\
\hline Ultrassom & 03 & $70 \%$ \\
\hline Pós-parto & 07 & \\
\hline Conhecimento do conceito de microcefalia & & $100 \%$ \\
\hline Sim & 10 & \\
\hline
\end{tabular}

Fonte: próprios autores, 2019.

Aos pais e responsáveis, foi perguntado o que seria possível pensar e realizar para melhorar o atendimento no Centro de Referência da Criança e, consequentemente, à criança com diagnóstico de microcefalia.

A tabela 5 mostra qual o nível de satisfação dos usuários do Sistema de Saúde para com o atendimento aos pacientes com microcefalia. Foi possível investigar sobre os tipos de atendimentos de saúde ofertados às crianças com microcefalia. Foram citados atendimentos com profissionais como: enfermeiro, médico, fisioterapeuta, terapeuta ocupacional e fonoaudiólogos. A pergunta disparadora possibilitou a opção por mais de uma resposta, pois apresentou respostas distribuídas em múltiplas escolhas, em que o participante poderia optar por mais de uma resposta, o que justifica a soma percentual com resultado superior a $100 \%$.

Quando questionado sobre o serviço de atendimento que necessitava de melhorias, o usuário informou que deveria haver melhorias no atendimento da fisioterapia, com $35 \%$; serviço de enfermagem, com $21 \%$; e psicologia, $13 \%$. No entanto, é importante frisar que o profissional de psicologia no momento da coleta de dados da pesquisa não compunha a equipe de saúde multiprofissional desse Centro de Referência da Criança.

Isso impulsiona a ressaltar a importância do profissional de psicologia como parte integrante da equipe de avaliação e acompanhamento das crianças com diagnóstico de microcefalia, para prestar suporte psicológico durante todo o acompanhamento, seja da mãe ou de toda a família.

De acordo com Sturmer (2011), o sistema de saúde deve ser um ambiente acolhedor que proporcione ao usuário a sensação de bem-estar e satisfação durante todo seu atendimento. Porém, a humanização é o ponto primordial para a satisfação do cliente, visto que o profissional de saúde deve observar o paciente de forma completa, buscando sempre solucionar a problemática e oferecendo a melhor assistência. 
É importante ressaltar que a equipe multidisciplinar é peça-chave para o bom desenvolvimento do portador de microcefalia, visto que essa equipe é treinada e capacitada para proporcionar melhoria na qualidade de vida da criança. Terapias podem realizar melhorias nas habilidades da criança, ocasionando assim maior conforto e satisfação para o cliente e sua família (LOTUFO, 2016).

Tabela 5 - Avaliação do serviço, profissionais de saúde que prestam atendimento e tipos de serviços a serem melhorados no Centro de Referência à Saúde da Criança

\begin{tabular}{lll}
\hline Descrição & Universo & $\%$ \\
\hline Profissionais de saúde envolvidos nos atendimentos & & \\
\hline Enfermeiro & 09 & $21 \%$ \\
\hline Médico & 10 & $23 \%$ \\
\hline Fisioterapeuta & 08 & $19 \%$ \\
\hline Terapeuta Ocupacional & 07 & $16 \%$ \\
\hline Fonoaudiólogo & 09 & $21 \%$ \\
\hline Melhorias no atendimento de: & & \\
\hline Enfermeiro & 05 & $21 \%$ \\
\hline Médico & 01 & $4,3 \%$ \\
\hline Psicólogo & 04 & $13 \%$ \\
\hline Fisioterapeuta & 08 & $35 \%$ \\
\hline Terapeuta Ocupacional & 01 & $4,3 \%$ \\
\hline Fonoaudiólogo & 04 & $13 \%$ \\
\hline
\end{tabular}

Fonte: próprios autores, 2019.

\section{CONCLUSÃO}

Diante do universo estudado no período de pesquisa, fica demonstrado um resultado insatisfatório relacionado ao atendimento dos profissionais de saúde que atuam no Centro de Referência em Saúde da Criança de Santarém, pois, apesar dos colaboradores realizarem o atendimento a crianças com microcefalia, eles relatam a necessidade de melhoria na estrutura física do atendimento e a promoção de capacitações para os profissionais que atuam diretamente com a criança portadora de microcefalia.

A importância no programa de atualização aos profissionais de saúde sobre a temática da microcefalia visa melhorar o atendimento ao infante com a doença e sua família e, consequentemente, promover melhor qualidade de vida, haja vista que a microcefalia não tem cura, sendo possível realizar apenas o tratamento paliativo, com o propósito de minimizar o sofrimento da criança e sua família.

Diante dos resultados analisados, foi possível perceber que o objetivo da pesquisa foi alcançado de maneira satisfatória. A melhoria no atendimento deve ser vista como fator primordial dentro das unidades de saúde, haja vista que a microcefalia é uma patologia incapacitante que, quando não tratada adequadamente, promove graves sofrimentos à criança e à família.

A equipe de enfermagem é de suma importância no tratamento e acompanhamento da criança com microcefalia. 0 enfermeiro é peça principal dentro da equipe multidisciplinar, pois é o responsável por levar todas as orientações à família em relação à doença. Por isso, é necessário que o profissional de enfermagem seja atualizado e capacitado frequentemente acerca da microcefalia, para que o atendimento de saúde seja realizado de forma qualificada, visando sempre à busca da qualidade de vida da criança, da gestante e de sua família. 


\section{REFERÊNCIAS}

AMARAL, F.E et al. Qualidade do pré-natal: uma comparação entre gestantes atendidas na Faculdade de Medicina de Barbacena e na Universidade Federal de Juiz de Fora. Clin Biomed Res 2016; 36(3).

BARCELLOS, M. B. A; RAPOPORT, A. Concepcção do professional do atendimento ocupacional sobre a deficiência. Rev pós-graduacao: desafios contemporâneos. v 1, n 1, jun/2014.

BARINI, R. et al. Desempenho da Ultra-sonografia Pré-natal no Diagnóstico de Cromossomopatias Fetais em Serviço Terciário. Rev. Bras. Ginecol. Obstet. [online]. 2002, vol.24, n.2, pp.121-127. ISSN 0100-7203. Disponível em: http://dx.doi.org/10.1590/S0100-72032002000200008. Acesso em: 05 abr. 2017.

BRASIL. M. S. Secretaria de Atenção à Saúde. Protocolo de atenção à saúde e resposta à ocorrência de microcefalia relacionada à infecção pelo vírus zika [recurso eletrônico] / Ministério da Saúde, Secretaria de Atenção à Saúde. - Brasília: Ministério da Saúde, 2016.

COSTA, E. S. et al. Alterações fisiológicas na percepção de mulheres durante a gestação. Rev. Rene. Fortaleza, v. 11, n. 2, p. 86-93, abr./jun.2010.

FILHO, E. A. F. et al. Toxoplasmose aguda: Estudo de frequência, taxa de transmissão vertical e relação entre os testes diagnósticos materno-fetais em gestantes em estado da Região Centro-Oeste do Brasil. Rev Bras Ginecol Obstet. 2005; 27 (8): 442-9.

FISCHER, C. Estado garante atendimento ao bebê com microcefalia associada ao Zika Vírus. 2016. Disponível em: http://www.saude.pa.gov.br/?p=3003. Acesso em: 10 maio 2017.

JANSEN, R. De zika a rubéola: as doenças que podem causar más-formações em fetos. Disponível em: http://www.bbc.com/portuguese/noticias/2016/02/160202_doencas_microcefalia_zika_lab_rj. Acesso em: 05 fev. 2016.

LOTUFO, P. A. Microcefalia, zika vírus e a soberania da observação clínica. Rev diagnóstico e tratamento . vol 21. Edição 1, 2016.

MENEZES, H. L. S. et al. Zika vírus associado a microcefalia. Rev pato Tocantins. V 3. N2, 2016.

NADER, P. R. A.; Características de abortamentos atendidos em uma maternidade pública do Município da Serra - ES. Rev. bras. epidemiol. vol.10 n.4 São Paulo. Dec. 2007.

NORBERT, A. A. F. et al. A importância da estimulação na microcefalia. XXIV Seminário de Iniciação Científica. Salão do Conhecimento. Unijuí, 2016.

PERUCCI, G. Dengue, microcefalia e Zika vírus: Como evitar? Disponível em: http://www.lersaude.com. br/dengue-microcefalia-e-zika-viruscomo-evitar/. Acesso em: 18 mar. 2016.

SANTOS, R.S; DIAS I. M. V. Refletindo sobre a malformação congênita. Rev Bras Enferm 2005set-out; 58(5):592-6.

SANTOS, A. L. et al. Assistência pré-natal: satisfação e expectativas. Rev. Rene, vol. 11, Número Especial, 2010. p. 61-71. 
STURMER, L. A importância do atendimento humanizado nos serviços de saúde em nobres, MT. XVI Seminário Interintitucional Pesquisa e Extensão. IX mostra de extensão, 2011.

SOUZA, F. S. et al. Incidência de malformação congênita e atenção em saúde nas instituições de referência. Rev. Rene, Fortaleza, v. 11, n. 4, p. 29-37, out./dez. 2010.

OLIANI, A. H. et al. Ultrassonografia morfológica do primeiro trimestre-passo a passo. Rev brasileira de ultrassonografia: RBUS/ Sociedade Brasileira de Ultrassonografia- vol.18. Goiania: Contato Comunicação, 2015. 